\title{
ECOLOGICAL INTERFACE DESIGN FOR ANAESTHESIA MONITORING
}

\author{
Marcus Watson', W. John Russell ${ }^{2}$, and Penelope Sanderson' \\ ${ }^{1} S$ winbume Computer-Human Interaction Laboratory \\ School of Information Technology \\ Swinburne University of Technology \\ John Street, Hawthom, Victoria 3122 \\ hutp://www.it.swin.edu.au/schil \\ ${ }^{2}$ Department of Anaesthesia and Intensive Care \\ Royal Adelaide Hospital \\ North Terrace \\ Adelaide, SA 5000
}

\begin{abstract}
The operating theatre is a noisy place with many uninformative and redundant alarms. Using data from a recent observational study, we demonstrate that anaesthetists actively respond to only $3.4 \%$ of all audible alarms. We outline a range of possible solutions to the alarm problem. Ecological Interface Design (EID) helps to outline the requirements for an information environment for anaesthetists and to indicate the possible benefits of continuous auditory signals. Our observational data are then "reworked" to give an indication of possible benefits of a continuous auditory display. Finally we indicate steps we are taking to test these ideas empirically.
\end{abstract}

\section{KEY WORDS}

Interface design, patient monitoring systems, alarm systems, cognitive engineering, ecological interface design

\section{INTRODUCTION}

Much of the literature on human interaction with medical equipment-such as information systems and devices in the operating room (OR) - has focused on critiques of existing devices (Cook \& Woods, 1996; Bogner, 1994). Less often have suggestions been made about the redesign of equipment and prototypes developed. However in the last few years, under the stimulus of Ecological Interface Design, concrete design suggestions and prototypes have emerged (Effken, Kim \& Shaw, 1997; Lin, Isla, Doniz, Harkness, Vicente \& Doyle, 1995; Sharp \& Helmicki, 1998). In this paper we outline a long-standing problem with unwanted and uninformative alarms in the OR and take the first step towards a new kind of solution.

When looking at how to design effective alarms in critical care environments, many researchers have applied findings from alarm studies in aviation and nuclear power plant environments (Cook \& Woods, 1996). However the anaesthesia and critical care environments pose distinct problems of their own. New technologies are continually being introduced in a piecemeal fashion rather than part of an integrated system. Moreover, the most important part of the sensed environment-the patient-is not a fixed engineered system, but instead has unique physiological characteristics that should be taken into account when determining what is normal and abnormal. Finally, alarms reflect the state of sensor technologies that have their own failure modes independent of patient status. In light of these factors we undertook an initial observational study to determine the problems and needs in OR alarm systems.

\section{BACKGROUND}

We studied anaesthetists' use of alarms in a series of observations at Royal Adelaide Hospital (RAH). Observations were over a two-week period and involved 42 operations varying in length, type and monitoring equipment used by anaesthetists. The focus was on detecting whether auditory alarms successfully directed an anaesthetist's attention to a problem with the patient or with the anaesthetic machine. We recorded the number of times an alarm limit was breached and the number of times auditory signals sounded. Furthermore any manipulation of the auditory alarm systems by the anaesthetists to monitor the patient (such as changing upper or lower limits, or using alarms as reminders to visually check trends) were recorded. Alarms were classified under several headings: physiological parameter indicated by the alarm (eg blood pressure, heart rate); type of alarm (lower or higher limit breached, noise in signal, equipment disconnection or warning); severity of the alarm; number of times the alarm sounded due to the incident (total number of auditory warnings for each alarm); phase of the operation in which the alarm occurred; and time. In addition the anaesthetist's response to each alarm signal was observed and coded.

As Figure 1 shows, $35.4 \%$ of the alarm sounds reflected the initiation or first sounding of a specific alarm and the remaining $64.6 \%$ were "run-on" alarms, simply re-signalling the initial alarm. Only $3.4 \%$ of all alarm sounds caused the anaesthetist to actively make adjustments to change patient state-only $5.3 \%$ of the initial alarms and $1.9 \%$ of the run-on alarm sounds led to responses. Of the 125 alarm limits breached during induction, 20 cases (16\%) led to a response; of the 232 alarm limits breached during maintenance, $29(12.5 \%)$ led to a response; and of the 124 alarm limits breached during emergence, only one led to a response $(0.8 \%)$.

Anaesthetists are well aware of the failure of alarms to indicate useful information. Typical comments made by the anaesthetists in our sample include the following: 
- "I know the NIBP alarm [blood pressure high] is going to go off again; [however] it takes a little while for these drugs to take effect and the pressure is already dropping"

- "Not much I can do about that alarm [ECG noise alarm]. Every time the surgeon uses the diathermy it'll go off. If I silence it I might miss an alarm that is important"

- "When the disconnect alarms are going, you normally have your hands full with the patient coming around. You know they [alarms] are unimportant, so you have to ignore them".

As Woods (1995) has noted, alarms often go off in parallel or in cascades, compounding difficult situations rather than helping them. The RAH data showed this both in critical incidents and in routine activity. The former is best illustrated in one operation where the patient's heart rate, blood pressure, oxygen saturation and respiration all started to decline rapidly. This was accompanied by alarms from $\mathrm{SpO}_{2}, \mathrm{NIBP}$ and the gas monitoring system. The anaesthetist was searching the monitor for information before the alarms sounded, having already noticed the downward trend. The anaesthetist's finger was ready to silence the alarms before they even went off. After this the focus was on the patient and several visual alarms flashed for other monitoring devices that had reached alarm limits, but since they were now silenced they were missed by the anaesthetist as he dealt with the patient. There were further frequent cases of disconnect warnings for two or more of $\mathrm{SpO}_{2}$, ECG, NIBP and ETCO $\mathrm{E}_{2}$ that all started in the same minute and lasted for 10 to 20 repeats while the anaesthetic staff attended to the patient.

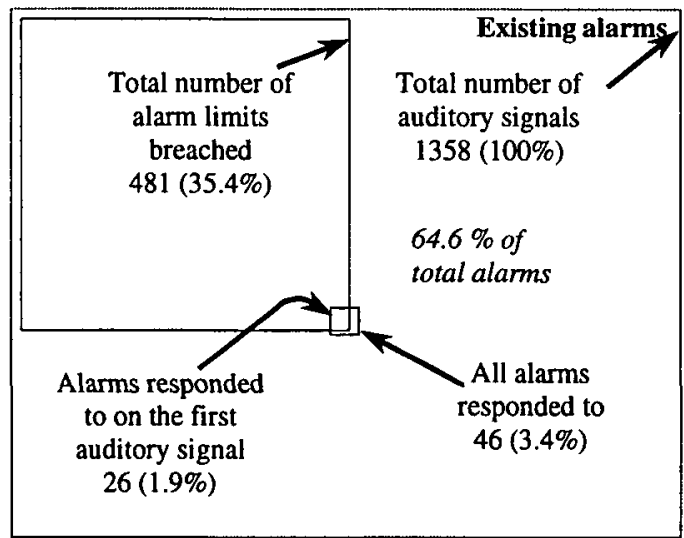

Figure 1. Alarms breached, auditory events, and anaesthetists' responses

\section{DESIGN GOALS}

Our goal is to contribute to the design of a more effective information environment about patient state. The quantitative and qualitative data reviewed indicate just some of the problems with current alarm systems in the operating theatre. Clearly, a more effective alarm management environment would have to achieve the following:

- Decrease confusion about which alarm went off that currently forces anaesthetist to check on monitor

- Decrease the number of uninformative alarms that sound, decreasing noise stress

- Decrease the density of alarms during critical events

- Increase the proportion of clinically useful alarms over "nuisance" alarms

- Increase anaesthetist's situational awareness and monitoring of higher-order patient state variables.

Table 1 provides a range of possible solutions that have been suggested at various points by researchers and clinicians interested in alarms in operating theatre environments. The solutions include suppressing "run on" alarms, suppressing some alarms at specific phases (such as ECG disconnect alarms at emergence), using earcons, using a continuous auditory display, developing intelligent alarm management, and using a heads-up display. As Table 1 makes clear, all approaches have advantages and disadvantages. As will be seen, the solution we explore in this paper is the idea of using a continuous auditory display for key parameters. This approach has been in place for some years since the advent of pulse oximetry. The pulse oximeter is a peg-like device that clips to a patient's finger, ear or toe and measures oxygen saturation in arterial blood as well as heart rate. The result is displayed continuously as a series of beeps where the rate indicates heart rate and the pitch of each beep indicates oxygen saturation, or $\mathrm{SpO} 2$. We are exploring the possibility of extending the concept of continuous auditory displays to other continuously sensed parameters. Ecological Interface Design is the conceptual framework we have chosen to guide this work. 


\section{EID APPROACH}

EID is an approach to interface design that is based in Rasmussen's Cognitive Work Analysis (Rasmussen, Pejtersen, \& Goodstein, 1994; Vicente \& Rasmussen, 1992; Reising \& Sanderson, 1998). The goal of EID is to engineer an information "ecology" around the human operator that is as easy to understand and as simple to control as the natural ecology in which we have evolved. The steps to achieve this are as follows:

1. Analyse the work domain in its first principles.

2. Analyse the control tasks that must be performed for the work domain to achieve its functional purpose.

3. Map critical properties of the work domain onto visual or auditory displays in a way that best supports the execution of control tasks (often termed "semantic mapping").

\begin{tabular}{|l|l|l|}
\hline \multicolumn{1}{|c|}{ Suggestion } & \multicolumn{1}{|c|}{ Advantages } & \multicolumn{1}{c|}{ Disadvantages } \\
\hline $\begin{array}{l}\text { Suppress "run- } \\
\text { on" alarms }\end{array}$ & $\begin{array}{l}\text { Reduction of noise in OR (64.6\% } \\
\text { fewer alarms in RAH data) }\end{array}$ & $\begin{array}{l}\text { Anaesthetist may forget to check if preoccupied. Design } \\
\text { challenge to tell when "run-on" alarm finished and new } \\
\text { incident starts. }\end{array}$ \\
\hline $\begin{array}{l}\text { Suppress selected } \\
\text { alarms at certain } \\
\text { phases }\end{array}$ & Annoying alarms removed. & Danger of false negatives. \\
\hline Use "earcons" & Type and origin of alarm discernible. & $\begin{array}{l}\text { Still the same amount of noise. Information still } \\
\text { "separated". }\end{array}$ \\
\hline $\begin{array}{l}\text { Use redundant } \\
\text { signals for single } \\
\text { measure }\end{array}$ & Artifact-based alarms reduced. & $\begin{array}{l}\text { Not available for all parameters. Anaesthetist unsure of } \\
\text { accuracy of measurement due to the variation between } \\
\text { different devices. }\end{array}$ \\
\hline $\begin{array}{l}\text { Use continuous } \\
\text { auditory displays }\end{array}$ & $\begin{array}{l}\text { Reduces the \# of alarms needed. } \\
\text { Keeps the anaesthetist "in-the-loop" }\end{array}$ & $\begin{array}{l}\text { Continuous noise. Acoustic design challenge. Requires a } \\
\text { reevaluation with alarm noise. Only limited use possible. }\end{array}$ \\
\hline $\begin{array}{l}\text { Intelligent alarm } \\
\text { management }\end{array}$ & $\begin{array}{l}\text { Hypothesises higher order state. } \\
\text { Reduces low-level nuisance alarms. }\end{array}$ & $\begin{array}{l}\text { Degree of human trust is an issue. Reduction of human } \\
\text { "in-the-loop" an issue. }\end{array}$ \\
\hline $\begin{array}{l}\text { Head-up displays } \\
\text { Reduces the \# of alarms needed. } \\
\text { Keeps the anaesthetist "in-the-loop" }\end{array}$ & $\begin{array}{l}\text { Visual cues may be inadequate to attract anaesthetist } \\
\text { attention. Discomfort of head mounting for some.. }\end{array}$ \\
\hline
\end{tabular}

Table 1. Alarm management strategies: advantages and disadvantages

A key component of the anaesthesia work domain is the human body in normal, compromised, and deranged states. Figure 2 shows a work domain analysis (WDA) of some anatomical structures and physiological functions of the human body that are most relevant for anaesthesia. For any node in the hierarchy, nodes above it say "why" the structure or function is there, and nodes below it say "how" the function is achieved. Compromised or even deranged states emerge from abnormalities in the elements, processes, and functions noted in the analysis. Superimposed on the analysis are circles indicating the kind of information that is picked up by key sensors in the operating theatre.

The control tasks are all the activities that must be carried out for safe, effective anaesthesia. The anaesthetist's role is to support cell perfusion (oxygenation via maintaining airway, breathing, and circulation) while inducing effective anaesthesia (an appropriate balance of pain relief, muscle relaxation, and sleep). This must be achieved with a safe induction (often including intubating the patient and running invasive lines) and a safe emergence (often including extubation and return to unassisted ventilation). Normal physiological functions are maintained, compromised functioning compensated for, and deranged states managed with the tools and drugs at the anaesthetist's disposal.

Semantic mapping involves revealing the properties, constraints, and boundaries of the work domain in a way that makes a direct connection with the control tasks to be performed. The "affordances"-or possibilities for action-in the work domain should be shown. The human operator should not have to laboriously infer what is happening from low-level data, but should instead be able to maintain a constant level of situational awareness about the patient's physiological state. Hitherto, most discussion about semantic mapping has dealt with visual displays (Buttigieg \& Sanderson, 1991; Bennett, Nagy, \& Flach, 1997; Hansen, 1995) and relatively little with auditory displays (Gaver, 1993; Gaver, Smith, \& O'Shea, 1991). It is notable that the most comprehensive treatment of the ecological approach to human-machine systems (Flach, Hancock, Caird \& Vicente, 1995) does not include a chapter on the auditory environment. For human-machine systems designers, auditory displays are almost exclusively auditory alarm systems. Although there is a substantial tradition of work into the discriminability and management of alarms (Stanton, 1994) there is very little on the continuous display of system state through the auditory modality.

However, Woods (1995) presents an alternative view of the possibilities for the auditory environment in continuous process environments. He argues that an alarm system should be seen as an "agent" that attempts to direct the attention of the human operator. An auditory signal should help maintain a continual level of awareness in the 
operator, at what Woods calls a "pre-attentive" level. The display should be capable of reorienting attention towards a changed or changing parameter before an alarm sounds so that the alarm is unnecessary or when the alarm does sound, the operator already knows what is happening with that parameter.

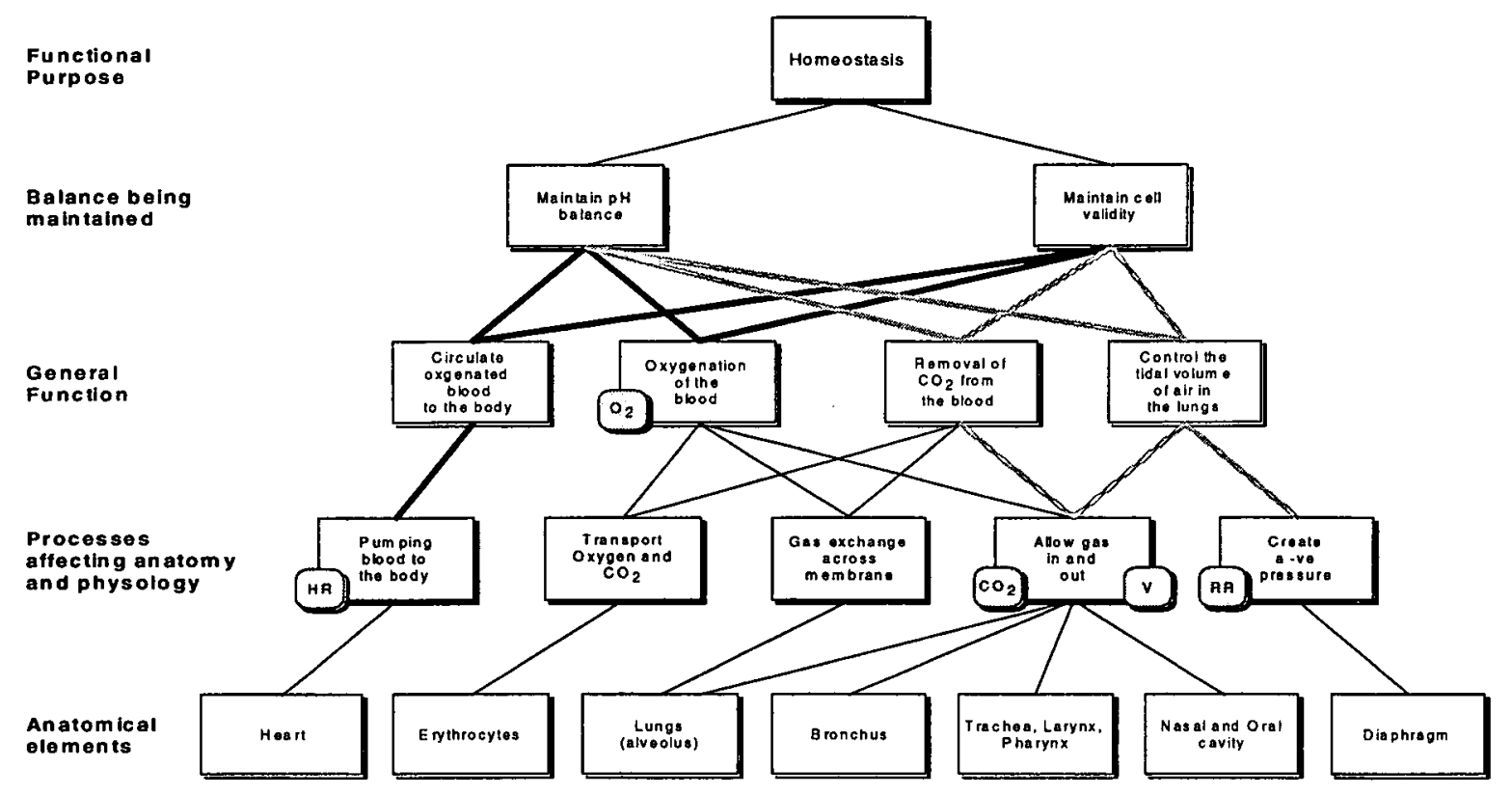

Figure 2. WDA of cardiovascular and respiratory parameters

In the domain of anaesthesia, the auditory pulse oximetry display is a paradigmatic case: If the pitch changes (indicating dropping oxygen saturation) or the auditory event rate changes (indicating a change in heart rate), anaesthetists often turn to check the digital readout on the monitor before an alarm limit is reached. Pulse oximetry has been shown to be highly successful at preventing incidents. Runciman, Webb, Barker, \& Currie (1993) have reported that of the 1256 incidents in the Australian Incident Monitoring Study (AIMS), pulse oximetry detected the largest proportion of evolving incidents. Figure 2 positions the two parameters picked up by pulse oximetry on the work domain analysis of the human body. Oxygenation of the blood is a general function inferred from saturation at one anatomical point. Heart rate is a physical process picked up by the device. Together they provide important information about physiological balances being maintained.

In our work we are exploring the feasibility of extending continuous auditory display to other systems-especially the respiratory system. The importance of continuously monitoring cardiac and respiratory rhythm has been advocated for over 80 years (Runciman et al, 1993). The value of such monitoring is strongly corroborated in the AIMS study. As noted, of the 1258 monitor-related incidents, $27 \%$ incidents were first picked up by pulse oximetry (device most often first to detect an incident) and 24\% by capnography, or CO2 monitoring (device next most often to detect an incident). Figure 2 positions three key respiratory parameters: end tidal (or expired) $\mathrm{CO} 2$ as picked up by capnography, respiration rate, and end tidal volume on the work domain analysis. The three parameters are sensed as processes rather than as functions. However, the WDA makes it clear how they combine to indicate general physiological functions and balances.

\section{REWORK OF RAH DATA}

Continuous auditory display of key parameters should, in principle, remove the anaesthetist's dependence on limitbased alarms for information about those parameters if the anaesthetist happens not to be watching trends on the monitor. We conducted a "what if" analysis with the RAH data to see how many alarm sounds would have been eliminated if limit alarms were removed and a continuous auditory display used. We first looked at the reduction in alarms if limit alarms in pulse oximetry were suppressed and then if both pulse oximetry and respiratory alarms were suppressed. We hasten to point out that, at this stage, we do not recommend that limit alarms should be suppressed. Continuous auditory displays may, however, allow limit alarms to indicate only more extreme deviations from the norm than is currently the case. Moreover, the analysis that follows does not necessarily reflect what would happen if continuous auditory displays were introduced and limit alarms suppressed, since such an arrangement would have a reactive effect and would possibly lead to a quite different monitoring strategy by the anaesthetist.

The right hand figure in Figure 3 shows the expected reduction in auditory alarms across the 42 operations when pulse oximetry and respiratory systems have their limit alarms removed. The total number of alarm sounds drops to $53 \%$ of the original value and the number of alarm incidents drops to $37 \%$ of the original value. It is important to 
note that seven alarms coded as "useful" that the anaesthetist responded to and three further "useful" alarms that the anaesthetist did not respond to would not have sounded under this policy. However there would still have been a continuous auditory signal carrying the information. It is possible that a continuous (ongoing) auditory signal with no limit alarm may be safer than the 10 episodes (out of 42 operations) in which the main monitor alarms were completely silenced for long tracts of the operation. The general reduction in alarms particularly the confusing and "cry wolf" alarms, can be expected to lead to greater attention by the anaesthetist to the alarms that remain (Bliss et al., 1995).

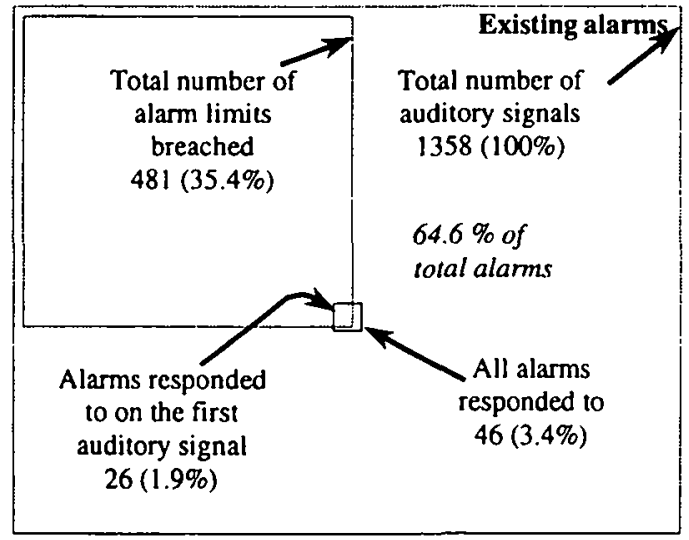

Propsed auditory alarms

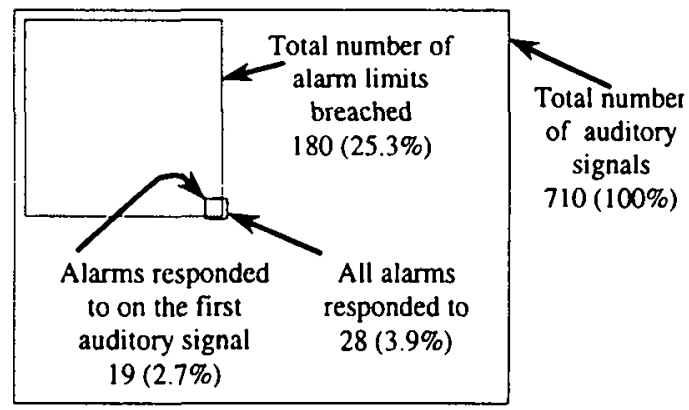

Figure 3. Auditory events with suppressed $\mathrm{CO}_{2}$ and respiratory alarms

Figure 4 shows the rate per minute of alarm limits breached and all alarm sounds (1) as currently observed in the RAH data, (2) if there were no pulse oximetry limit alarms and (3) if there were neither pulse oximetry nor respiration limit alarms. Pulse oximetry and respiration alarms contribute equally to the rate at which alarm limits re breached and the rate per minute of all alarm sounds. The persistence of larger alarm rates during emergence is due to the very high number of ECG and NIBP disconnect signals that occurred during emergence in most operations.

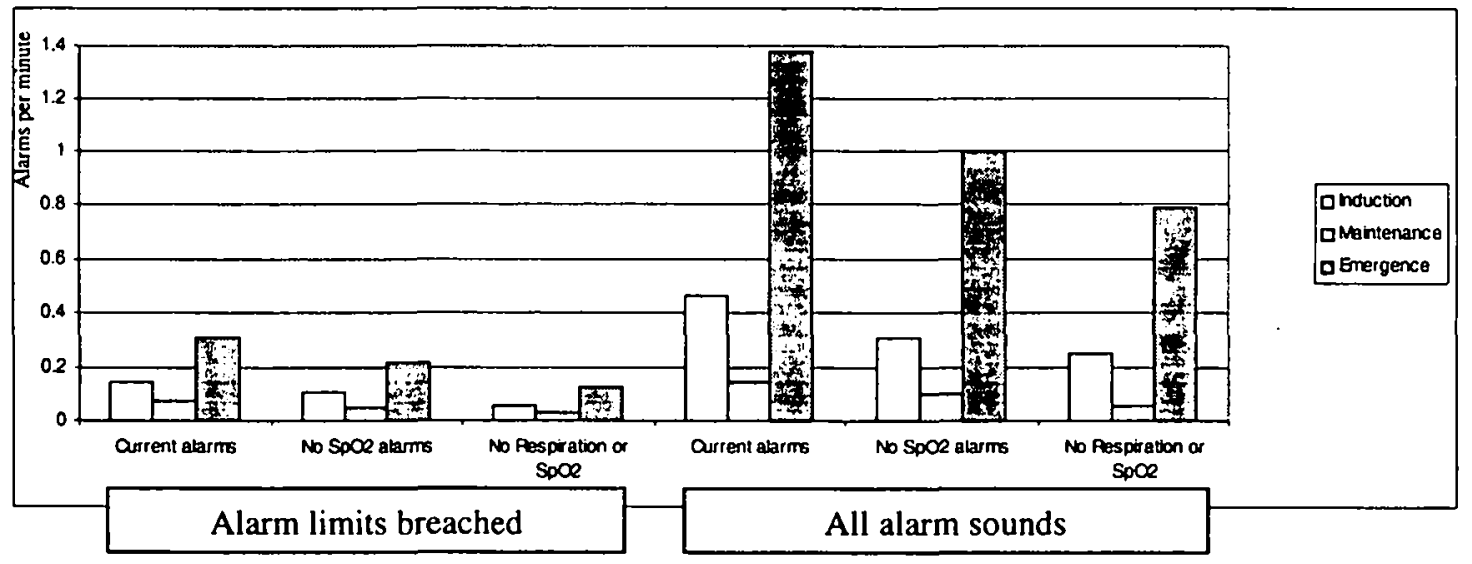

Figure 4. Reduction in auditory events over operative phases

Overall, the above "what if" analysis establishes that there may be some value in downplaying limit alarms in favour of continuous auditory monitoring. Respiratory parameters are clearly pivotal, as the WDA in Figure 2 shows as well as the AIMS study data. The challenge for an ecological approach will be designing the continuous auditory display for end tidal $\mathrm{CO} 2$, respiration rate and possibly also end tidal volume so that it is easy for the anaesthetist to extract the higher order-properties of patient state, as shown in Figure 2 . For this task we seek a configural auditory display (Buttigieg \& Sanderson, 1991; Bennett et al., 1997). Moreover, the sound signal chosen for a continuous auditory display for respiratory model must coexist successfully-in terms of psychoacoustics and auditory aesthetics - with pulse oximetry sounds. Combining the insights of Woods (1995) with an important EID goal of making boundaries of safe operation very evident, continuous auditory display of physiological parameters should become more potentially attention-attracting as boundaries of safe regions are approached and bypassed. 


\section{CURRENT AND FUTURE WORK}

We are developing continuous auditory alarms in a small-scale but versatile anaesthesia simulation environmentthe BODY ${ }^{\mathrm{TM}}$ Anesthesia Simulator from Advanced Simulation Corporation. BODY provides us with a testbed for research and development into alarm management systems. BODY contains a dynamic physiological and pharmacological model, on top of which we are constructing a fully end-user configurable research environment (BODY + ) that allows us to develop and to empirically evaluate alternative alarm management systems. Any alarm management system that performs well in laboratory tests must be evaluated in a full-scale anaesthesia simulators for there to be a basic level of confidence in its potential effectiveness for the OR.

\section{ACKNOWLEDGEMENTS}

This work was supported by an ARC Small Grant to the third author. We thank Royal Adelaide Hospital for permission to conduct the study and RAH anaesthetists for their participation. We also thank Tim Purtill and Matthew Trentini for programming support and Malcolm Boyd for advice about the BODY Anesthesia Simulation.

\section{REFERENCES}

Bennett, K., Nagy, A. \& Flach, J. (1997). Visual displays. In G. Salvendy (Ed.), Handbook of Human Factors and Ergonomics (2nd edition). New York: Wiley.

Bliss, J. P., Gilson, R. D., \& Deaton, J. E. (1995). Human probability matching behavior in response to alarms of varying reliability. Ergonomics, 38, 2300-2313.

Bogner, M. S. (1994). Human error in medicine. Hillsdale, NJ: LEA.

Buttigieg, M. A., \& Sanderson, P. M. (1991). Emergent features in visual display design for two types of failure detection tasks. Human Factors, 33, 631-651.

Cook, R. I. \& Woods, D. D. (1996). Adapting to new technology in the operating room. Human Factors, 38, 593613.

Effken, J. A., Kim, N.-G., \& Shaw, R. E. (1997). Making the constraints visible: testing the ecological approach to interface design. Ergonomics, 40, 1-27.

Flach, J., Hancock, P., Caird, J., \& Vicente, K. (1995). Global perspectives on the ecology of human-machine systems (Volume 1). Hillsdale, NJ: LEA.

Gaver, W. (1993). How in the world do we hear? Explorations in ecological acoustics. Ecological Psychology, 5 , 283-313.

Gaver, W., Smith, R., \& O'Shea, T. (1991). Effecrive sounds in complex systems: The ARKola simulation sound. Proceedings of the ACM CHI'91 Conference on Human Factors in Computing Systems. Association for Computing Machinery. pp. 85-90.

Hansen, J. P. (1995). Representation of system invariants by optical invariants in configural displays for process control. In P. Hancock, J. Flach, J. Caird, \& K. Vicente (Eds.), Local applications of the ecological approach to human-machine systems (Volume 2). Hillsdale, NJ: LEA

Lin, L., Isla, R., Doniz, K., Harkness, H., Vicente, K., \& Doyle, J. (1995). Analysis, redesign, and evaluation of a patient-controlled analgesia machine interface. Proceedings of the Human Factors and Ergonomics Society $3^{\text {th }}$ Annual Meeting. San Diego, CA. October 9-13.

Rasmussen, J., Pejtersen, A. \& Goodstein, L. P. (1994). Cognitive systems engineering. New York: Wiley Interscience.

Reising, D. V., \& Sanderson, P. M. (1998). Designing displays under ecological interface design: Towards operationalizing semantic mapping Proceedings of the 42nd Annual Meeting of the Human Factors and Ergonomics Society. Chicago, IL. 4-7 October.

Runciman, W. B., Webb, R. K., Barker, L., \& Currie, M. (1993). The pulse oximeter: Applications and limitations-An analysis of 2000 incident reports. Anesthesia and Intensive Care, 21, 543-550.

Sharp, T., \& Helmicki, A. (1998). The application of the ecological interface design approach to neonatal intensive care medicine. Proceedings of the Human Factors and Ergonomics Society $\mathbf{4 2}^{\text {nd }}$ Annual Meeting. Chicago, IL. October 5-9. Santa Monica, CA: HFES.

Stanton, N. A. (1994). Human factors in alarm design. London: Taylor \& Francis.

Vicente, K., \& Rasmussen, J. (1992). Ecological interface design: Theoretical foundations. IEEE Transactions on Systems, Man, and Cybernetics, SMC-22, 589-606.

Woods, D. D. (1995). The alarm problem and directed attention in dynamic fault management. Ergonomics, 38, 2371-2394. 